\title{
Open fistulectomy with sphincter fixation for anal fistula
}

\author{
Tatsuya Abe $^{1 *}$, Masao Kunimoto ${ }^{1}$, Yoshikazu Hachiro ${ }^{1}$, Yoshiaki Ebisawa ${ }^{1}$, Houhei Hishiyama ${ }^{1}$, \\ Seishu Abe $^{2}$ \\ ${ }^{1}$ Department of Proctology, Kunimoto Hospital, Asahikawa, Japan \\ ${ }^{2}$ Department of Plastic Surgery, Kunimoto Hospital, Asahikawa, Japan \\ Email: *t-abe@cf6.so-net.ne.jp
}

Received 1 June 2013; revised 1 July 2013; accepted 20 July 2013

Copyright (C) 2013 Tatsuya Abe et al. This is an open access article distributed under the Creative Commons Attribution License, which permits unrestricted use, distribution, and reproduction in any medium, provided the original work is properly cited.

\begin{abstract}
Purpose: This study aimed to report clinical data and recurrence rates in patients with anterolateral low fistulas who underwent open fistulectomy with sphincter fixation. Methods: The study group consisted of 133 consecutive patients with anterolateral, low intersphincteric, or low trans-sphincteric fistulas who had undergone open fistulectomy with sphincter fixation between January 2006 and December 2010. This procedure involves complete removal of the fistula tract by incision of anal sphincters, followed by fixation of the sphincter muscles. Results: Success was achieved in $127(95.5 \%)$ patients with a median follow-up time of 12 months. Anal fistula recurred in 4 cases (3\%). Non-healing fistula with persistent anal discharge developed in 2 patients. Maximal resting pressure, but not maximal squeeze pressure, was significantly decreased after surgery. Five patients $(4 \%)$ developed temporary anal incontinence after surgery. Conclusions: Open fistulectomy with sphincter fixation was effective for the management of patients with anterolateral low fistula in this study. The high success rate suggests that this procedure is a reasonable option in this group of patients.
\end{abstract}

Keywords: Anal Fistula; Fistulectomy; Sphincter Fixation; Fecal Incontinence

\section{INTRODUCTION}

Anal fistula is a devastating problem that most commonly occurs in healthy subjects, with cryptoglandular infection being the most widely accepted etiological factor [1]. On the basis of their anatomical course relative to the sphincter complex, cryptoglandular fistulas are categorized as intersphincteric, trans-sphincteric, suprasphinc-

"Corresponding author. teric, and extrasphincteric types [2]. The most common type is intersphincteric, followed by transsphincteric [2, 3]. Anal fistulas can also be classified as simple or complex. The major type is simple, which includes low intersphincteric and low trans-sphincteric fistulas that cross $<30 \%$ of the external sphincter [4].

Most fistulas have been conventionally treated by fistulotomy or fistulectomy, which have both proven to be effective [5]. However, these techniques, even for simple fistulas, result in some form of incontinence in approximately $12 \%-39 \%$ of patients $[3,5,6]$. In most patients, the internal opening of the fistula is located along the posterior midline [7]. Fistulas located laterally are significantly associated with recurrence, and those located along the anterior midline are associated with incontinence $[3,8]$. Anterolateral localization results in greater postoperative anal deformity when fistulotomy or fistulectomy is used to excise the fistula [9]. Therefore, more attention is needed for the management of anterolateral fistulas.

Open fistulectomy with sphincter fixation (OFSF) is another option for the management of anterolateral fistulas. The procedure is based on the premise that removal of the chronic epithelialized tract will allow healing by secondary intention of healthier tissue, and sphincter fixation may result in less deformity and prevention of incontinence compared with leaving the sphincters divided. However, this is a more extensive procedure, and there is lack of support for it in the literature. The aim of this study was to evaluate clinical data and recurrence rates in patients with anterolateral fistulas who underwent OFSF.

\section{PATIENTS AND METHODS}

The study group consisted of 133 consecutive patients with anterolateral, low intersphincteric, or low transphincteric fistulas who had undergone OFSF between January 2006 and December 2010 at our institution. The median 
age of these 133 patients (122 males) was 42 years (range, 16 - 87 years). All patients presented with chronic fistula of the cryptoglandular origin. All had a history of previous perineal suppuration drained surgically or spontaneously. Fistula tracks and internal opening were clinically and endosonographically evaluated and were found in the anterior quadrant in 89 (67\%) and in the left or right lateral quadrant in 44 (33\%) patients.

OFSF was performed under caudal epidural anesthesia in the prone jackknife position with the buttocks taped widely apart. A suppository rather than an enema was used to empty the rectum before surgery. The external opening along with the fistula tract was circumscribed and dissected using an electric cautery or Metzenbaum scissors. Dissection was performed as deep into the sphincter as possible. The internal opening was excised using a Parks retracter to efface the anal canal. All tissues between the internal and external openings were cut open by sphincterotomy, and all of the fistula tract was removed. Both edges of the incised internal and external anal sphincter (IAS and EAS, respectively) muscles were slightly mobilized to ensure fixation without tension. Next, interrupted 3-0 poliglecaprone was used to suture the muscles to the base of the defect area after fistulectomy (Figure 1). A simple layer of interrupted 3-0 poliglecapronesutures was used to marsupialize the proximal edge of the anoderm to prevent postoperative bleeding. Distal anoderm and perianal skin were left open to facilitate drainage. Postoperatively, the patients were administered prophylactic oral antibiotics (cefaclor 750 $\mathrm{mg} /$ day) and oral analgesia (loxoprofen $180 \mathrm{mg} /$ day) for 3 days, and there were no dietary restrictions.

A $5 \mathrm{~mm}$ diameter, 1-channel, solid-state catheter with a microtipped transducer anorectalmanometry (ARM) system (P-31; Star Medical Co., Tokyo, Japan) was used to perform ARM before and 3 months after surgery. All patients were examined in the left lateral position with the hips flexed to $90^{\circ}$. The maximal resting pressure (MRP) and maximal squeeze pressure (MSP) were analyzed. Manometric data were expressed as means \pm stan-

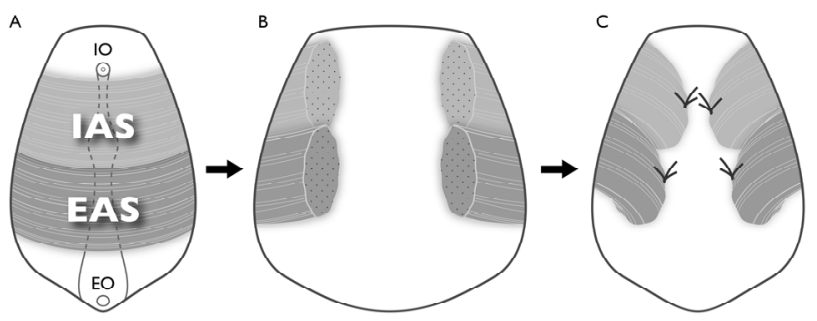

Figure 1. Detail of open fistulectomy with sphincter fixation. (A) IO = internal opening, EO = external opening, IAS = internal anal sphincter, EAS = external anal sphincter; (B) Sphincter division and total fistulectomy; (C) Both edges of the incised IAS and EAS were sutured to the base of the defect area after fistulectomy. dard deviations. Wilcoxon signed-rank test was used to test statistical significance. Statistical significance was set at $\mathrm{p}<0.05$.

This study was a retrospective review of existing clinical data prospectively collected on a hospital anal physiology unit computer database. The study was approved by the research and ethics committee of Kunimoto Hospital, and all patients gave written informed consent.

\section{RESULTS}

The median operative time was 21 min (range, 10 - 42 min). There were no postoperative deaths. Postoperative bleeding occurred in 2 patients (1.5\%) and required stitching of the bleeding area, which was sited at the edge of the wound. No significant dehiscence of the sphincter fixation occurred. Of the 133 patients, 127 (95.5\%) healed completely and did not require any further surgical treatment, with a median follow-up of 12 months (range, 2 - 62 months). Anal fistula recurred in 4 cases (3\%). Recurrence in these patients was observed at $3,6,8$, and 12 months. Non-healing fistula with persistent anal discharge developed in 2 patients (1.5\%); 1 of them with persistent symptoms had an identifiable fistula tract connecting the anal canal to skin, and the external opening failed to close, with infected granulation in the other patient.

Seventy two (54\%) of the 133 patients underwent ARM before and after surgery. MRP was significantly decreased after surgery, but not MSP (Table 1). Clinically, 5 patients (4\%) developed temporary anal incontinence after surgery. Solid stool incontinence was not present, but liquid stool incontinence and flatus were observed in 2 and 3 males, respectively; all had recovered in $<6$ months.

\section{DISCUSSION}

The overall rate of fistula persistence or recurrence was $4.5 \%$ and temporary minor incontinence was $4 \%$, which showed the effectiveness of OFSF in the management of anterolateral low fistulas in these patients. The management of anal fistulas includes 3 main goals: to cure the fistula, to prevent or minimize recurrence, and to retain continence. Since OFSF allows recognition of the full length of an anal fistula via direct visualization enabled

Table 1. Changes in maximal resting and squeeze pressure $(\mathrm{n}=$ 72).

\begin{tabular}{lccc}
\hline & Preoperative & Postoperative & p value \\
\hline MRP (mmHg) & $77.6 \pm 18.0$ & $65.8 \pm 18.1$ & $<0.05$ \\
MSP (mmHg) & $239 \pm 86.8$ & $249 \pm 99.4$ & 0.41 \\
\hline
\end{tabular}

$\mathrm{MRP}=$ maximal resting pressure; $\mathrm{MSP}=$ maximal squeeze pressure. 
by sphincter division, removal can certainly be a primary focus, which eliminates the risk of missing secondary tracts and allows healing by secondary intention of healthier tissue. Moreover, divided sphincters are fixed to prevent reduction in anal resting tone. We have also used this technique successfully to manage complex fistulas, but the present study was limited to a more common group, simple fistulas.

Both fistulotomy and fistulectomy have long been accepted as the gold standard for simple fistulas. Although fistulotomy has been associated with success rates of $92 \%-97 \%$, the procedure will result in some form of incontinence even for simple fistulas in approximately $12 \%-39 \%$ of patients $[3,5,6]$.

Both fistulotomy and fistulectomy leaves the unepithelialized wound opened, which may cause undesired pain and complications, such as bleeding and suppuration. Marsupialization of the unepithelialized wound was introduced to provide the benefit of shortened healing times and improved continence by minimizing anal deformity [10]. The addition of marsupialization also has been associated with less postoperative pain and bleeding [11]. However, this procedure leaves the sphincters divided, and Pescatori et al. [11] did not observe any superiority of marsupialization with respect to incontinence rates.

Fistulectomy and immediate sphincter repair for low fistulas, described by Parkash et al. [12], aim to eradicate infection and to anatomically reconstruct the muscular defect. However, very few studies have been published on this technique. Dehiscence of sphincteroplasty is the most fearful complication of this technique and is responsible for its infrequent use [13]. Roig et al. [14] treated 75 patients in whom most of the fistulas were complex (69\%) by fistulectomy and end-to-end reconstruction of the disrupted EAS. Five recurrences in total have been described (6.7\%), and the postoperative incontinence rate was 21\%. Perez et al. [13] included 16 patients with recurrent complex fistulas in whom fistulotomy was performed with overlapping repair of the disrupted EAS. Two (25\%) of 8 fully continent patients developed incontinence for watery stools and flatus, which gave a recurrence rate of $6.3 \%$ (1 patient). By overlapping the muscle, the area in contact is increased and rupture of the sutures may be more unlikely. However, when the width of overlap is too long, the anal canal may narrow and drainage worsen.

The strengths of sphincter fixation, our original method, are as follows: 1) since both edges of the sphincters are sutured separately, the tension on the sutures can be reduced by half relative to that on end-to-end sutures; 2) extensive dissection of sphincter muscles is not needed to achieve overlap; 3) the anal canal does not become narrow as can occur with overlap. The edges of the di- vided sphincter muscles will separate rapidly when they are not at all repaired. Even if they are fixed in the original position, continence will be fully maintainable. In fact, it was found that sphincter fixation was better preserved for MSP 3 months after surgery. Although MRP was significantly reduced, the extent was only $15 \%$. Clinically, 5 patients (4\%) developed temporary incontinence for liquids and gas, and all of them had recovered in $<6$ months.

\section{CONCLUSION}

The true advantages of the OFSF procedure may not be clear until larger prospective randomized studies are conducted. However, considering the current reported data, we believe that OFSF can achieve complete removal of fistulas safely and easily with minimal risk of postoperative incontinence and low recurrence rates.

\section{REFERENCES}

[1] Parks, A.G. (1961) Pathogenesis and treatment of fistula-in-ano. British Medical Journal, 1, 463-469. doi:10.1136/bmj.1.5224.463

[2] Parks, A.G., Gordon, P.H. and Hardcastle, J.D. (1976) A classification of fistula-in ano. British Journal of Surgery, 63, 1-12. doi:10.1002/bjs.1800630102

[3] Garcia-Aguilar, J., Belmonte, C., Wong, W.D., et al. (1996) Anal fistula surgery. Factors associated with recurrence and incontinence. Disease of the Colon and Rectum, 39, 723-729. doi:10.1007/BF02054434

[4] Parks, A.G. and Stitz, R.W. (1976) The treatment of high fistula-in-ano. Disease of the Colon and Rectum, 19, 487499. doi:10.1007/BF02590941

[5] Davies, M., Harris, D. and Lohana, P. (2008) The surgical management of fistula-in-ano in a specialist colorectal unit. International Journal of Colorectal Disease, 23, 833-838. doi:10.1007/s00384-008-0444-x

[6] Roig, J.V., Jordan, J., Garcia-Armengol, J., et al. (2009) Change in anorectal morphologic and functional parameters after fistula-in-ano surgery. Disease of the Colon and Rectum, 52, 1462-1469. doi:10.1007/DCR.0b013e3181a80e24

[7] Atkin, G.K., Martins, J., Tozer, P., et al. (2011) For many high anal fistulas, lay open is still a good option. Techniques in Coloproctology, 15, 143-150. doi:10.1007/s10151-011-0676-6

[8] Steele, S.R., Kumar, R., Feingold, D.L., et al. (2011) Practice parameters for the management of perianal abscess and fistula-in-ano. Disease of the Colon and Rectum, 54, 1465-1474. doi:10.1097/DCR.0b013e31823122b3

[9] Iwadare, J. (2000) Sphincter-preserving techniques for anal fistulas in Japan. Disease of the Colon and Rectum, 43, S69-S77. doi:10.1007/BF02237229

[10] Ho, Y.H., Tan, M., Leong, A.F.P.K., et al. (1998) Marsupialization of fistulotomy wounds improves healing: A randomized controlled trial. British Journal of Surgery, 
85, 105-107. doi:10.1046/j.1365-2168.1998.00529.x

[11] Pescatori, M., Ayabaca, S.M., Cafaro, D., et al. (2005) Marsupialization of fistulotomy and fistulectomy wounds improves healing and decreases bleeding: A randomized controlled trial. Colorectal Disease, 8, 11-14. doi:10.1111/j.1463-1318.2005.00835.x

[12] Parkash, S., Lakshmiratan, V. and Gajendran, V. (1985) Fistula-in-ano: Treatment by fistulectomy, primary closure and reconstruction. Australian and New Zealand Journal of Surgery, 55, 23-27.

doi:10.1111/j.1445-2197.1985.tb00849.x
[13] Perez, F., Arroyo, A., Serrano, P., et al. (2006) Prospective clinical and manometric study of fistulotomy with primary sphincter reconstruction in the management of recurrent complex fistula-in-ano. International Journal of Colorectal Disease, 21, 522-526. doi:10.1007/s00384-005-0045-X

[14] Roig, J.V., Garcia-Armengol, J., Jordan, J.C., et al. (2009) Fistulectomy and sphincteric reconstruction for complex cryptgrandular fistulas. Colorectal Disease, 12, e145e152. 